\title{
Deep Core muon neutrino rate and anisotropy by mixing and CPT violation
}

\author{
Fargion D., ${ }^{1,2}$ D'Armiento D., ${ }^{2}$ \\ ${ }^{1}$ INFN, Rome University 1, Italy \\ ${ }^{2}$ Physics Department, Rome Univ. 1 Ple A.Moro 2, 00185, Rome, Italy
}

June 9, 2018

\begin{abstract}
Neutrinos are allowed to mix and to oscillate among their flavor. Muon and tau in particular oscillate at largest values. Last Minos experiment claimed [16] possible difference among their matter and anti-matter masses, leading to a first violation of the most believed CPT symmetry. Isotropically born atmospheric muon neutrino at $E_{\nu_{\mu}} \simeq 20-80 \mathrm{GeV}$, while up-going, they might be partially suppressed by mixing in analogy to historical SuperKamiokande muon neutrino disappearance into tau, leading to large scale anisotropy signals. Here we show an independent muon rate foreseen in Deep Core based on observed SK signals extrapolated to DeepCore mass and its surrounding. Our rate prediction partially differ from previous ones. The $\nu_{\mu}, \overline{\nu_{\mu}}$ disappearance into $\nu_{\tau}, \overline{\nu_{\tau}}$ is leading to a $\mu, \bar{\mu}$ anisotropy in vertical up-going muon track: in particular along channel $3-5$ we expect a huge rate (tens of thousand of events) of neutral current events, charged current electron and inclined crossing muons. Moreover at channel $6-9$ we expect a severe suppression of the rate due to muon disappearance (in CPT conserved frame). Such an anisotropy might be partially tested by two-three string detection at $E_{\overline{\nu_{\mu}}} \geq 45$. A CPT violation may induce a more remarkable suppression of vertical up-going tracks because of larger $\overline{\nu_{\mu}}$ reduction for $E_{\overline{\nu_{\mu}}} \geq 35$.
\end{abstract}

\section{Introduction}

The neutrino are very complex particles indeed. Their three light neutrino flavors mix in a complex way described by a matrix born only in last few decades [14, [20],[21]. Their presence maybe recorded in small kiloton detector or larger (like SK and Super Kamiokande 22 kiloton) ones.In largest size detectors as Icecube the higher characteristic $\nu_{\mu}, \overline{\nu_{\mu}} \mathrm{TeV}$ energy do not oscillate much and they do not exhibit the negligible oscillation along our narrow Earth. However the new born Deep Core, while tuning to few tens or even a few $\mathrm{GeV} \nu_{\mu}, \overline{\nu_{\mu}}$ energy, may hold memory of the $\nu_{\mu}, \overline{\nu_{\mu}}$ disappearance into tau. Many have foreseen the $\nu_{\mu}, \overline{\nu_{\mu}}$ disappearance in Deep Core [11] [17]. We did use their prediction to calibrate the eventual CPT violation influence into their future rate [9]. Here we review these predictions and now we reconsider our preliminary estimate, based on Super Kamiokande ones [4], estimates that partially disagree with the previous results [11][17][12] as well as [10]. Deep Core, is a new telescope or better a counter event blurred at low energies (below $E_{\nu_{\mu}} \leq 30 \mathrm{GeV}$ ) because muons are tracing tracks mostly projected along one string: the inner cone within $\sim 30^{\circ}$ may contain any neutrino arrival direction 
around the string axis azimuth angle. At higher energy $E_{\nu_{\mu}} \geq 45 \mathrm{GeV}$ the muon track, if inclined, may intersect two different strings leading to a much accurate (a few degree) angular resolution. Therefore the DeepCore may test different energy regions at different degree of resolution. Moreover most of the very inclined (respect the vertical) muons (below $E_{\nu_{\mu}} \leq 30 \mathrm{GeV}$ ) may intersect briefly the one string leading to a few (four-five) detector optical module (DOM) signal, in a very short timing clustering. This mean that most of the inclined events may accumulate, as a noise, into the a few channel region that is at the same time the deposit of ten (or tens) GeV shower, mostly Neutral Current, (NC), event by $\nu_{\mu}, \overline{\nu_{\mu}}, \nu_{\tau}, \overline{\nu_{\tau}}$ and Charged Current, $(\mathrm{CC})$, and $\mathrm{NC}$ due to electrons by $\nu_{e}, \overline{\nu_{e}}$. These few channel signals may also record rare (nearly a thousand) tau appearance. However the previous noise will make difficult to discover tau appearance, while muon disappearance is still viable.

\subsection{SuperKamiokande rate versus DeepCore}

The simplest way to estimate the Deep Core muon track and possible tau appearance (or better, the muon disappearance) has been shown by Icecube MC simulation [12], as described in figure 1, with our additional CPT violation expected influence[9]. However we show here an independent derivation of the expected Deep Core rate, based on SuperKamiokande one [1]. There are four main contribute to SK upgoing muons: Fully contained event (FC), born inside and decayed inside SK; partially contained event (PC), born inside but escaping outside the SK volume; the Upward Stopping Muons, born outside but decayed inside the detector; the upward through muon, just born outside, crossing and decaying again at external volume. Some care have been taken into account for the last Upward Stopping Muons and upward through muon: the SK location is deeply surrounded by mountain rock, while Deep Core is within much less dense ice. Therefore we suppressed the two last rates by the density ratio $(\simeq 2.6)$ to calibrate the expected rate in DeepCore, amplified by extrapolated volume ratio $\left(\frac{V_{D e e p C o r e}}{22 k T}\right)$ versus Deep Core one at each energy range. Indeed the Deep Core volume is variable with the muon energy values due to photodetector thresholds and muon Cherenkov luminosity. We considered here the preliminary Deep Core effective volume variability following last Icecube articles [6], [12], [17, [22]. Our result is described in the right side of figure 113 in linear scale along the channel number. We assumed an averaged neutrino muon and anti-muon energy conversion, their length projected along the string at spread angle of $\theta \simeq 30^{\circ}$. The total event number derived by simplest SK-DeepCore translation is huge: $N \simeq 97.800$. Most of these events are not vertical but inclined. Therefore assuming a vertical beaming solid cone suppression (also to reconcile with Deep Core total expected rate) we selected only those events within a cone angle $\pm 33^{\circ}$, obtaining a fraction $(1-\cos \theta \simeq 0.16)$ of the total rate, in this way now compatible with Deep Core preliminary global expectation $N \simeq 16.000$.

\section{$1.2 \quad$ Rates and anisotropy}

The calibrated muon rate figure 3 shows in grouped channel graph number, the rate that we foresee following SK within a narrow vertical axis along each string. These prediction do not overlap with previous one. In particular as we did mention we foresee a huge rate of inclined events whose NC produce shower observable by $3-4-5$ channel: this very rough estimate is based on the $\mathrm{NC}$, and electron CC,NC shower : they are well above $20.000 \mathrm{NC}\left(\right.$ for $\nu_{\mu} \nu_{\tau}$ ) with additional thousands of shower by $\mathrm{CC}$ and $\mathrm{NC}$ for $\nu_{e}$ and their antiparticles. The inclined tracks by nearly horizontal muons will excite the vertical string with a characteristic arrival time much shorter than any vertical shower event. Indeed the time difference in arrival for spherical shower along a string (each DOM at $7 \mathrm{~m}$ separation) is nearly $\Delta t_{0} \simeq t_{0}=h / c=23 n s$; by triangulation any horizontal muon tracks and its Cherenkov cone will record a quite shorter delay $\Delta t_{0} \simeq t_{0} \cdot\left(1-\frac{1}{\cos \left(\theta_{C}\right) \cdot n_{i c e}}\right) \simeq 0.08 t_{0}=1.84 \mathrm{~ns}$. Therefore this cluster of event, nearly coincident in time, might be a key test to calibrate the muon event rate in wide solid angle and possibly to meter the event rate at each channel. 

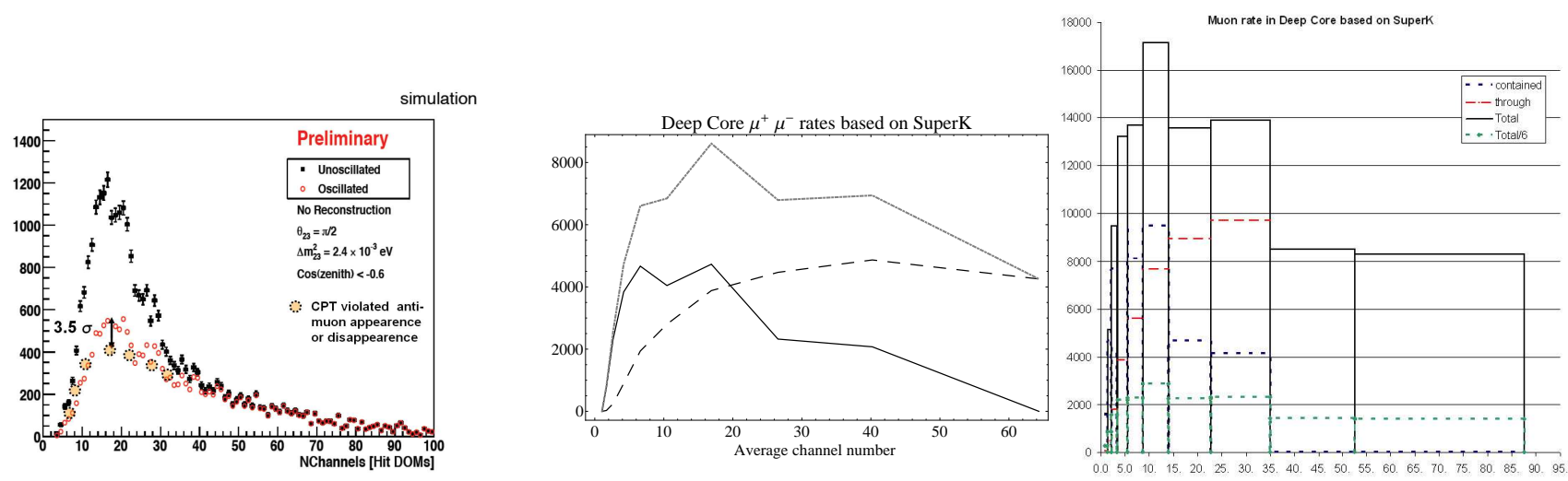

Figure 1: Left: The expected event rate following [6],[12], of atmospheric muon neutrinos whose tracks are detected along the photo-tubes (DOM) channel. The expected $\left(\nu_{\mu} \rightarrow \nu_{\tau}\right)$ and the anti-neutrino muon $\left(\bar{\nu}_{\mu} \rightarrow \bar{\nu}_{\tau}\right)$ induce a suppression. Our prediction for CPT violation, following [9] is also shown. The SK event rate in Deep Core extrapolated from SK for each different nature (FC,PC,Upward stopping, Through going) are shown in the center : dashed curve describe the upgoing stopping and upward through events; the FC and PC are described by lower continuous curve. The rate is much larger (16 times) probably because DeepCore is selecting only very vertical tracks, within $33^{\circ}$ from string axis, as shown in a normalized rate offered in figure 3 .

Oscillation probability for an initial ratio

$\bar{v}_{e}: \bar{v}_{\mu}: \bar{v}_{\tau}=0: 1: 0 \quad(<\mathrm{E}>\approx 50 \mathrm{GeV})$
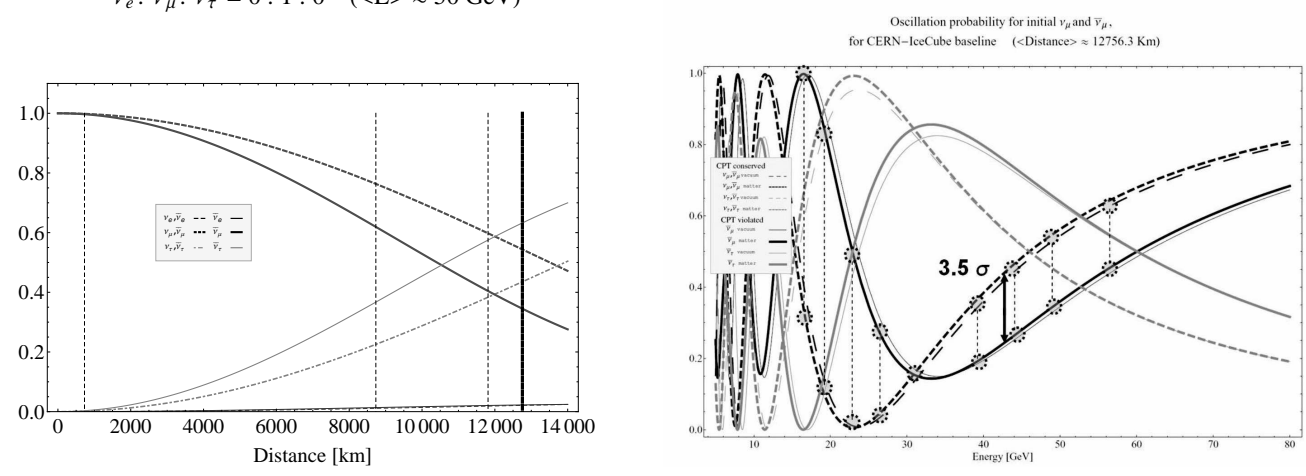

Figure 2: Left: In a CPT conserved (dashed lines) or in CPT violated case (continuous curve) scenario the neutrino conversion $\left(\nu_{\mu} \rightarrow \nu_{\tau}\right)$ and the anti-neutrino muon $\left(\bar{\nu}_{\mu} \rightarrow \bar{\nu}_{\tau}\right)$, as well as survival probability for given $50 \mathrm{GeV}$ energy at different distances [9]. The thick vertical line shows the Earth diameter, the dashed ones the Cern-Opera,SK,icecube distances. The same oscillation in energy function and at earth diameter distance, in CPT conserved (dashed line) and CPT violation (continuous line) scenario reflects additional suppression of muon survival $\left(\bar{\nu}_{\mu} \rightarrow \bar{\nu}_{\mu}\right)$ at energies $E_{\nu} \geq 44 \mathrm{GeV}$. 


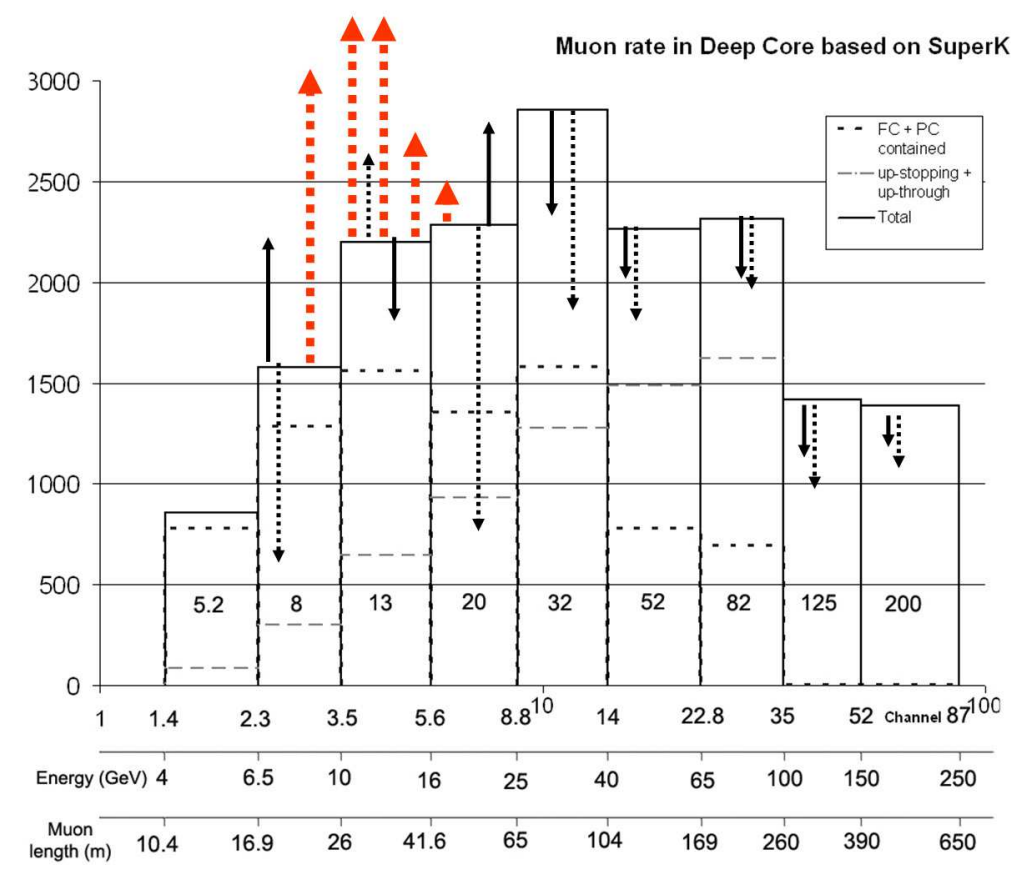

Figure 3: The expected event rate at each muon (and anti-muon averaged) energy band, its corresponding muon (and anti-muon averaged) length, its corresponding channel number projected by a $\cos (\pi-\theta)$ factor; $\theta \simeq 33^{\circ}$. The rate are modulated by large red arrows standing for nearly one o two tens of thousand noise event, by a continuous black arrow due to CPT violated influence, by a broken line due to CPT conserved muon tau disappearance. The influence of dashed arrow (muons) is twice larger than anti-muon ones because the twice larger neutrino versus antineutrino cross section. See Fig. 4.
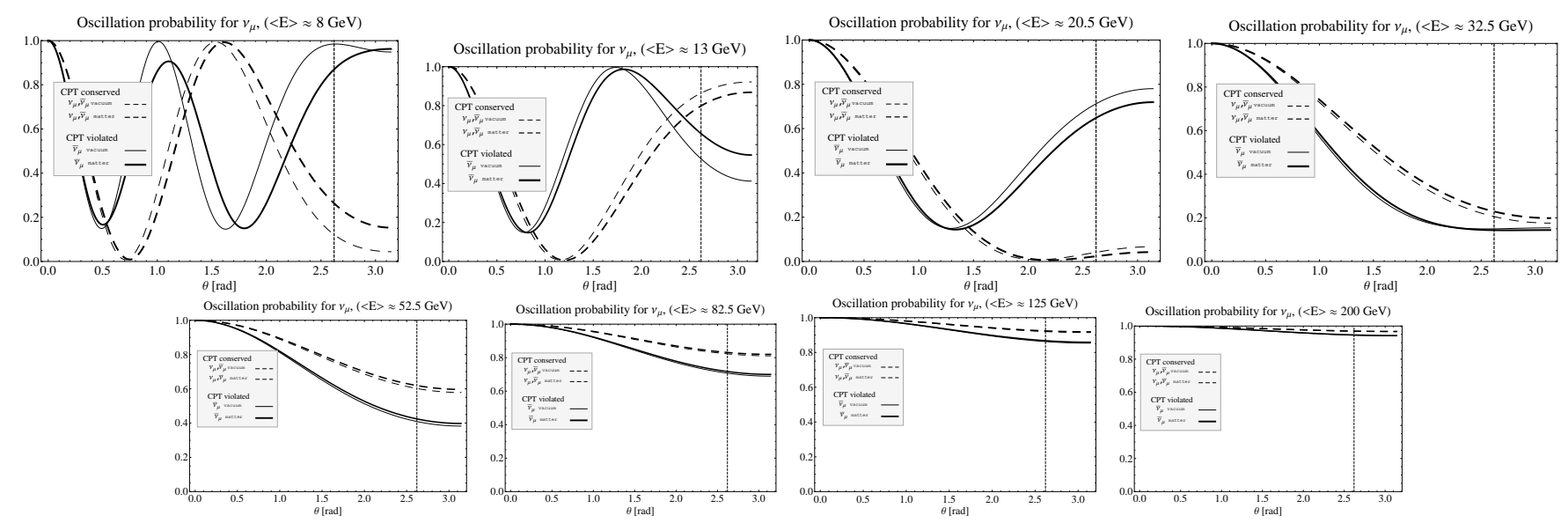

Figure 4: Eight different energy windows (corresponding to SK rate estimate above) for muon rates as a function of the arrival direction angle. Note the different suppression or enhancement of the oscillation in CPT violated (continuous) or conserved case (dashed) lines at vertical or quasi vertical arrival direction. These nearly vertical regions are above the vertical dashed line $(\pi-\theta \geq 2.6 \mathrm{rad}$ corresponding to $\theta \leq 33^{\circ}$ ) shown in each figure. The vertical downward North Pole points at $\theta \simeq \pi$ rad. The averaged energy label on the top of each figure corresponds to the channel region in figure above Fig. 3 


\section{Conclusions}

We did show the rate of atmospheric muon neutrinos along Deep Core string channels with some comments on the expected anisotropy. The main results are: a) a very sharp peaked morphology in muon rate, namely a huge noise rate in low range $3-6$ channel (ten thousand or above event a year) followed by b), a deep minimum along channel 7-9 due to muon disappearance (contrasted partially by eventual CPT violation), whose rate maybe below 2000 event a year. c) A global decay and suppression of described events Fig (3) in range above 10-50, all along each channel, because of muon disappearance and additional anti-muon CPT violated suppression, by the vertical muon tracks that are more wide and they suffer larger disappearance leading to more anisotropic behavior respect to the averaged SK rates. The present rate estimate differ from the most known ones [6],10,20; however the CPT violation influence foreseen in our previous paper [9] plays the same role: to reduce the common muon survival, making more anti-tau appearance, from channel above $\simeq 13$. The strong modulation by CPT violation at low channel $(3-6)$ number, is quite remarkable, but it is nevertheless useless because a huge noise pollution by $\mathrm{NC}$, electron $\mathrm{CC}, \mathrm{NC}$ and nearly horizontal muon traces.

\section{References}

[1] Abbasi R. et al. (IceCube Collaboration), arXiv:1010.3980v1.

[2] Aguilar J. A. for the IceCube Collaboration, arXiv:1010.6263.

[3] Akhmedov E., Phys. Scripta T121 (2005) 65-7; arXiv:hep-ph/0412029v2

[4] Ashie,Y. PHYSICAL REVIEW D 71,(2005) 112005;. arXiv:hep-ex/0501064

[5] Cabibbo N., Unitary Symmetry and Leptonic Decays, Phys. Rev. Lett. 10 (1963) 531-533.

[6] Cowen D., Journal of Physics: Conference Series, TeV Particle Astrophysics II Workshop 60 (2007) $227-230$.

[7] Kodama K. et al. (DONUT Collaboration), Observation of tau neutrino interactions, Phys. Lett. B 504 (2001) 218; doi:10.1016/S0370-2693(01)00307-0.

[8] Fargion D.,Astrophys.J.570:909-925,2002; . arXiv:astro-ph/9704205; Fargion D. et. al, Astrophys. J. 613 (2004) 1285-1301.

[9] Fargion D., D’Armiento D., Desiati P., Paggi P., arXiv:1012.3245;

[10] Giordano G., Mena O., Mocioiu I. arXiv:1004.3519v1

[11] Gandhi R. et al., Earth matter effects at very long baselines and the neutrino mass hierarchy, Phys. Rev. D 73 (2006).

[12] Grant D., Koskinen J., and Rott C. for the IceCube collaboration, Proc. of the 31st ICRC, Lodtz, Poland, 2009.

[13] Jeong Y. S. and Reno M. H., Phys. Rev. D 82 (2010) 033010.

[14] Maki Z., Nakagawa M., and Sakata S., Remarks on the Unified Model of Elementary Particles, Prog. Theor. Phys. 28 (1962) 870; doi:10.1143/PTP.28.870.

[15] Mikheev S. P. and Smirnov A. Y., Sov. J. Nucl. Phys. 42 (1985) 913-917. 
[16] MINOS Collaboration, website, http://www-numi.fnal.gov/PublicInfo/forscientists.html.

[17] Montaruli T., IceCube Collaboration, Proc. of CRIS 2010 Conference, Catania, Sep. 2010

[18] Nakamura K. et al. (Particle Data Group), J. Phys. G 37, (2010) 075021.

[19] Perl M. L. et al., Phys. Rev. Lett. 35 (1975) 1489.

[20] Pontecorvo B., Mesonium and anti-mesonium, Zh. Eksp. Teor. Fiz. 33 (1957) 549-551; reproduced and translated in Sov. Phys. JETP 6 (1957) 429.

[21] Pontecorvo B., Neutrino Experiments and the Problem of Conservation of Leptonic Charge, Zh. Eksp. Teor. Fiz. 53 (1967) 1717; Sov. Phys. JETP 26 (1968) 984.

[22] Schulz O. (IceCube Collaboration), AIP Conf. Proc. 1085 (2009) 783; C. Wiebusch and f. t. I. Collaboration, arXiv:0907.2263.

[23] Shinji M. and Seong C. P., arXiv:1009.1251v2, 8 Sep 2010.

[24] Wiebusch C. for the IceCube Collaboration, Proceedings of the 31st ICRC, Lodz, Poland, July 2009.

[25] Wolfenstein L., Phys. Rev. D 17 (1978) 2369. 